\title{
Re: The Microbiome and Genitourinary Cancer: A Collaborative Review
}

\author{
Markowski MC1, Boorjian SA2, Burton JP3,8, Hahn NM1, Ingersoll MA4, Maleki Vareki S5,6, Pal SK7, Sfanos KS1,8,9 \\ 1Sidney Kimmel Comprehensive Cancer Center, Department of Oncology, Baltimore, USA \\ 2 Mayo Clinic, Rochester, Department of Urology, USA \\ ${ }^{3}$ Western Ontario University, Division of Urology, Department of Surgery, London, Canada \\ 4 Institut Pasteur, Department of Immunology, Paris, France \\ ${ }^{5}$ Cancer Research Laboratory Program, Lawson Health Research Institute, London, Canada \\ 6Western Ontario University Faculty of Medicine, Department of Oncology, London, Canada \\ 7 City of Hope Comprehensive Cancer Center, Department of Medical Oncology \& Therapeutics Research, Duarte, USA \\ 8Johns Hopkins University Faculty of Medicine, James Buchanan Brady Urological Institute, Department of Urology, Baltimore, USA \\ 9Johns Hopkins University Faculty of Medicine, Department of Pathology, Baltimore, USA
}

Eur Urol 2019;75:637-646 doi: 10.1016/j.eururo.2018.12.043.

\section{EDITORIAL COMMENT}

The role of the genitourinary microbiome in many diseases is a popular research topic. Some studies have demonstrated possible roles of the gastrointestinal microbiota in cancer treatment response. In this review, the authors summarized the evidences of the role of the genitourinary and gastrointestinal microbiome in genitourinary cancer initiation. Furthermore, the gastrointestinal microbiota can have an effect on drug metabolism. Genitourinary microbiota may be causative factors or cofactors in genitourinary malignancy. On the other hand, fecal microbiota transplant is an interesting approach towards increasing the efficacy of immunotherapy. Genitourinary tract infection is a risk factor for the development of certain malignancies, such as squamous cell bladder cancer following chronic parasitic infections, e.g. Schistosoma haematobium infection. The chronic inflammatory response may be a cofactor in driving carcinogenesis. The presence of some species of bacteria that can mediate the formation of carcinogenic $\mathrm{N}$-nitrosamines as part of the urinary microbiota may contribute to development of bladder cancer. Furthermore, Bacillus Calmette-Guerin (BCG) vaccine, developed from Mycobacterium bovis, is widely used in preventing recurrence of bladder cancer by direct bladder instillation. BCG induces a tumor-specific immune response, in part through the binding of BCG to fibronectin. Commensal microorganisms may potentially interact with BCG, influencing the development of immunity to bladder cancer. Genitourinary microbiata may present in focal regions of the prostate, and perhaps in regions associated with foci of acute or chronic inflammation or "fossilized" in prostatic corpora amylacea. These areas can be associated with the carcinogenesis of the prostate. The role of the microbiome in genitourinary cancer will be an important field in the near future for many research.

Fehmi NARTER, MD, PhD

๑Copyright 2019 by the Association of Urological Surgery / Journal of Urological Surgery published by Galenos Publishing House. 